
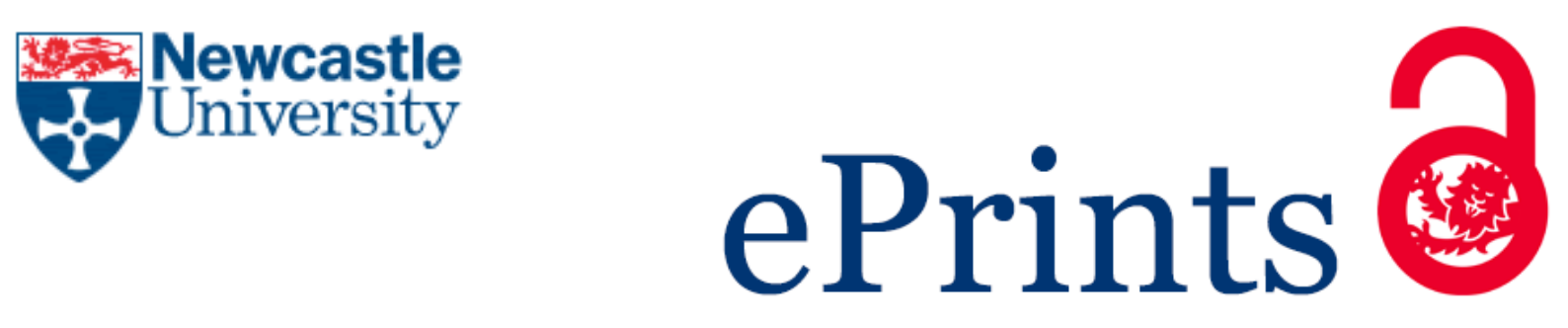

Marouf S, Bell M, Goodman P, Namdeo A, Neasham J.

Pervasive wireless sensors: A new monitoring tool for road traffic noise evaluation.

Applied Acoustics 2018, 135, 48-59.

\title{
Copyright:
}

(C) 2018. This manuscript version is made available under the CC-BY-NC-ND 4.0 license

DOI link to article:

https://doi.org/10.1016/j.apacoust.2018.01.017

Date deposited:

$08 / 02 / 2018$

Embargo release date:

02 February 2019

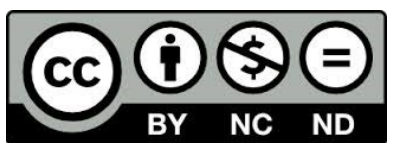

This work is licensed under a

Creative Commons Attribution-NonCommercial-NoDerivatives 4.0 International licence 


\title{
Pervasive Wireless Sensors:
}

\section{A New Monitoring Tool for Road Traffic Noise Evaluation}

\author{
Shadman Marouf* \\ s.s.marouf@ncl.ac.uk
}

\author{
Margaret Carol Bell ${ }^{*}$ \\ Professor \\ margaret.bell@ncl.ac.uk
}

\section{Paul Goodman*}

Research Associate

paul.goodman@ncl.ac.uk

\author{
Anil Namdeo* \\ Senior Lecturer \\ anil.namdeo@ncl.ac.uk \\ Jeffery Neasham ${ }^{\star \star}$ \\ Senior Lecturer \\ jeff.neasham@ncl.ac.uk
}

School of Engineering, Newcastle University, Cassie Building*, Stephenson Building ${ }^{\star *}$, Claremont Road, Newcastle upon Tyne NE1 7RU, United Kingdom

\begin{abstract}
Noise pollution is estimated to affect 170 million citizens in Europe, causing serious public health problems [1]. The World Health Organisation claims that at least one million healthy life years are lost per year in Europe due to road traffic noise [2]. Effective management of noise requires an understanding of its causes. This understanding is limited by traditional monitoring methods, which employ expensive equipment and are labour intensive. This paper presents the results of a comprehensive programme of correction and validation of a low-cost device referred to as an eMote for pervasive monitoring and is the first to quantify the accuracy of inexpensive noise systems that use microphones typically costing about one Euro.

Pervasive wireless noise sensors (eMotes) were validated by co-location with precision sound level meters in controlled indoor, and at roadside outdoor environments. Strong linear relationships between the eMotes and the precision systems, across a noise range between 55dBA and 94dBA were observed and exhibited consistent bias compared to the precision measurement. Therefore, a generic, corrective relationship was derived and validated in three contrasting outdoor traffic noise environments, employing both short-term attended, and long-term unattended measurements, which were carried out during day and/or evening and/or night periods.

The eMotes were shown to respond consistently to white and pink generated noise during the evaluation of the accuracy process, and the generic correction algorithm for white noise delivered better than 3dBA accuracy in comparison to precision data at a one-minute averaging resolution. The correction algorithm improved the concordance correlation coefficient (ccc) and coefficient of determination $\left(\mathrm{R}^{2}\right)$ of the eMote measurements against those of the precision instrument. Removal of short-duration, excessively loud events (e.g. sirens), which represented $2 \%$ of the total data, improved the ccc and $R^{2}$ values further typically to 0.74 and 0.60 respectively, which is considered good, given the limitations of the experimental procedure. The research provides scientific evidence that whilst not acceptable for compliance monitoring to standards for noise exposure, the eMote is a valuable system to screen for excessive exposure; to understand the causes of traffic related noise in urban areas; to provide an indication of the spatial and temporal variation in noise levels and the knowledge to design appropriate solutions, in turn this will lead to more effective abatement. The continued monitoring allows the impact to be quantified giving confidence that intervention measures are worthwhile, delivering added value compared to current measurement methods.
\end{abstract}


Applied Acoustics, 2018, Vol 135, pp 48-59

https://doi.org/10.1016/i.apacoust.2018.01.017

\section{Keywords:}

Noise pollution, Pervasive monitoring, Wireless sensors (eMotes), Road traffic noise

\section{Introduction}

Noise, or "unwanted sound", has been declared a pollutant since 1972 by the World Health Organization (WHO) $[3,4]$. Noise pollution is a major environmental problem in Western Europe, with traffic being one of the major sources. In the UK urban road traffic noise dominates, with approximately 12 million people exposed to levels sufficient to cause disturbance [5]. In EU countries, more than $40 \%$ of citizens have been estimated to be exposed to road traffic noise levels exceeding $55 \mathrm{dBA}$ during day time, whilst at night the figure is $30 \%$ [1]. It is estimated that 170 million citizens in Europe live in areas where noise levels cause serious health impacts during the daytime [6]. Whilst, the number of people exposed to noise pollution is much higher in developing countries, the long-term effects are the same in both [7]. There are several studies showing that environmental noise affects health and well-being through causing annoyance, sleep disturbance and cardiovascular diseases, such as hypertension and ischaemic heart disease [1]. The evidence presented by the WHO identifies the serious impact of environmental noise on public health throughout the world, estimating that at least one million healthy life years are lost per year in Europe due to road traffic noise [2]. The same report concludes that traffic noise annoys one in three individuals during the daytime and disturbs one in five at night.

These facts, along with growing evidence of the levels of noise and their health effects prompted the WHO in 1999 to publish guidelines for community noise [8]. These guidelines present noise threshold values and health effects when values exceed specific limits. For example, to protect the majority of residents from moderate and serious annoyance the day time outdoor noise LAeq Should not exceed 50 and 55dBA respectively, and the levels should be lower by 5 and 10dBA for evening and night period respectively. At night time [9] advocates that outdoor LAeq above 40dBA should be mitigated against suggesting $55 \mathrm{dBA}$ as an interim target for the countries where the achievement is not feasible in the short term. The first round of noise mapping was delivered in 2007 and the second round was due for submission in October 2012, but as yet in 2017 maps are not available to the general public.

Current noise maps are mostly created using noise prediction models [1], which are based mainly on data from transportation planning or traffic assignment models with composition, speed and flow estimates representing peak periods of typically 2 or 3 hours duration. These often fail to provide accurate estimates of noise pollution levels which need hourly levels over 24 hour periods of the day. If monitored data is available the input variables are usually averaged for each hour over the day across a measurement period but at one location on a link. Data is available for typically $50-100$ locations across a city. However, these isolated measurements are assumed to be representative of the entire length of road leading to noise estimates which fail to capture the variability of, and change in levels of noise along the length of a road and in close proximity to, and at, junctions.

Commonly used noise monitoring equipment enables highly reliable and precise noise measurements to be made, but measurements are often limited to few sites due to the high cost of instruments (up to 30,000 euros) [10]. Also, such measurements are labour intensive, especially when noise levels need to be measured at numerous points across a study area [11]. Because of security issues, precision monitors cannot be realistically deployed on the roadside for extended periods. Hence, data collection periods may be short, potentially introducing errors and biases caused by unrepresentative short-term effects. Murphy and King [1] claimed that at least 14 days of continuous noise monitoring is needed to give a useful description of the noise environment. Such assessment is expensive and impractical especially for cities with limited budgets [12]. Another issue with precision measurement is that, given limited equipment availability, monitoring can only ever capture noise levels at a few, discrete positions at a time, resulting in asynchronous noise readings at different locations [13]. High-precision monitoring may be required across large areas, with the number or distribution of monitoring points not matching available resources $[12,14]$. Noise level maps can only be verified and validated by the direct measurement of the spatial and temporal variations of noise levels across an urban area, but given the fact that precision sound level meters are so expensive and labour intensive, validation of maps is severely restricted to a few sites only.

In the last decade, to overcome the shortfalls of precision monitoring systems, scholars [10, 15-21], have researched alternative more cost effective and convenient approaches to monitoring noise. This led to the development of low cost pervasive sensor systems, that deploy wireless networks, to monitor noise along roads, at dwellings, or in sensitive locations. 
The UK Engineering and Physical Sciences Research Council (EPSRC) funded the project MESSAGE (Mobile Environmental Sensing System Across Grid Environments) which led to the development of pervasive sensor prototypes [18, 22]. These were commercialised as eMotes (electronic Mote) by Envirowatch Ltd.. In general, such acoustic networks consist of a number of autonomous, low cost, self-powered single processor sensors referred to as 'nodes' within a network. Other examples of pervasive sensor systems include: Tmote-Sky [19] CiNet [21, 23], IoT [24], Tml and RPi [20], ELECTRET1, ELECTRET4, TYPEII, MEMS1 [15] and CinetNoise sensor node [10]. The cost of an electret microphone used with the sensor systems typically ranges from one to a few hundred Euros or pounds sterling. Given their low cost, large numbers of sensor systems may be deployed over wide areas to monitor variation in road traffic noise spatially and temporally, enabling both realtime and long-term data collection, with a minimal amount of maintenance. The nodes within the network communicate using multi-hop routing protocols and typically provide the equivalent A-

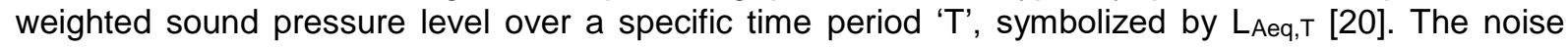
level data are transferred co-operatively from an individual node's memory to a central 'sink' node more often referred to as a 'gateway'. The latter acts as an external connector, which has an internal clock to provide an accurate time stamp to the data captured in the network, and sends the data wirelessly to a remote central server for data synchronisation and storage either using 3G/GPRS modem or WiFi connection. The server, or client systems, may provide additional facilities for the postprocessing and visualisation of the collected data.

Measurements of road traffic noise with different Wireless Sensor Network systems have been demonstrated and published in the literature [15, 16, 20,21,25]. Generally, the authors asserted the possibility to deploy the wireless noise sensors, on street furniture along roadsides. However, none of the published projects, except [15], have mentioned both long-term data collection along with colocation of the precision sound level meter, to check the accuracy of the measurements, considering the within-hour and day-to-day variability in noise levels from road traffic.

Van Renterghem et al. [15] deployed eight different types of microphones, ranging from low to high price in an outdoor environment for six months, to assess the microphone performance under different weather conditions. The microphones, installed on a bar at height equal to $1.7 \mathrm{~m}$, faced a busy trafficked viaduct at about $150 \mathrm{~m}$ distance. The average noise level was $65 \mathrm{dBA}$ during the day, and $50 \mathrm{dBA}$ at night. The study demonstrated the high correlation between (5 out of 7 ) inexpensive wireless microphones tested and the reference microphone. This was a Brüel and Kjær type 4189 microphone capsule, connected to the dedicated noise measurement hardware system, Brüel and Kjaer PULSE software system, with front end type 3560C. However, the study only derived the coefficient of determination, which measures how close the data are to the fitted regression line, but failed to report any departure from the true line with a slope and intercept equal to 1 and 0 respectively as was the case for Segura-Garcia et al. [20].

In the field trial [21] of five low cost sensors were deployed in a line perpendicular to a motorway, with 10 metres separation between the units. A precision Cesva SC-20c SLM was systematically colocated with each sensor in turn giving simultaneous 5 minute samples. The results showed on average a difference of less than 2dBA between the measured and reference levels. However, the measurement period of 5 minutes especially at a sampling rate of $1 \mathrm{~Hz}$, given the huge variability in the levels of road traffic noise prevailing during the day and night, is considered to be insufficient to appropriately evaluate the accuracy.

In 2009, the MESSAGE project designed, developed and deployed prototype low cost pervasive sensors at various sites in England (Leicester, Leeds, Gateshead and London) to measure noise, as well as air pollution (carbon monoxide, nitric oxide and nitrogen dioxide), temperature and humidity. A deployment of 50 wireless sensors was made in Leicester, UK, covering an area of approximately $1 \mathrm{~km}^{2}$, which included different types of roads and acoustic scenarios in the vicinity of a busy signalised junction. These early prototype eMotes were validated by co-location with a precision monitor. The results highlighted the consistency of measurement across all eMotes and insensitivity of the low cost microphones to short-term peak noise events, and to levels below 45dBA [26] and concluded that the accuracy of measurement was $~ 3 \mathrm{dBA}[25]$. However, the response of the microphone across the audible frequency range was not studied and no attempt was made to correct for the discovered

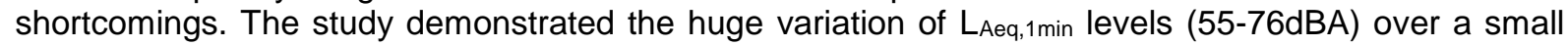
urban area caused by traffic, endorsing the value of the low cost sensor sampling at $5 \mathrm{~Hz}$ and at an averaging interval of one minute, in order to capture the variations in the probability density functions of urban noise [25]. 
The lack of systematic long term evaluation of the performance of inexpensive systems revealed by the literature, was the motivation of this research to explore further the functionality of the pervasive sensors. Their accuracy was investigated by comparing the eMote noise measurement with precision instruments, in controlled indoor and in-situ field conditions. Ways to improve the eMote accuracy were explored and subsequently the potential application of the wireless sensors to monitor traffic noise levels throughout the day, outdoors and examine further the eMote response when noise levels fall below the threshold of the detection range of microphone.

This section has provided background information on the sources, effects and problems with monitoring and assessing traffic noise pollution. It has highlighted the difficulties of collecting noise data using traditional precision systems and the potential benefits of low cost pervasive monitoring, provided the accuracy of measurement of the new technology is known and any systematic error identified can be automatically corrected. The next section will give more detail about the eMotes as an example of a low cost sensor. Section three presents the eMote evaluation carried out indoors to derive a generic self-correcting algorithm. Section four tests the algorithm in a heavy trafficked outdoor environment and presents the results from the validation procedure. Section five explores the reliability of these noise sensors and their suitability to monitor traffic noise over a range of urban areas. Finally, the paper concludes with a discussion of the accuracy of these wireless sensors and their suitability to monitor road traffic noise. Suggestions as to their potential use to calibrate and validate noise models to gain a better understanding of and develop a novel process of evaluating human exposure to traffic noise pollution are also made.

\section{2 eMotes (Pervasive Monitoring Devices)}

The key drivers in the design of the eMote device were cost, physical size, low power consumption and operation within a wireless network. The system uses ZigBee wireless communication [27], which allows the data to pass (hop) from one eMote to another when they are within an $80-100 \mathrm{~m}$ range. The system allows five hops between individual eMotes before reaching a gateway device, which allows communication with the central server. The gateway therefore needs to be located with line-of-sight to those eMotes at the end of a hop chain, to allow good communication links. The low power eMote circuitry, generates an envelope signal from the microphone with a smoothing time constant of $1 \mathrm{~s}$; samples this signal at $5 \mathrm{~Hz}$ and averages for 59s out of every minute. The eMotes are time synchronised to better than $100 \mu \mathrm{s}$ by beacon transmissions from the gateway node and simultaneously wake up their IEEE 802.15.4 (Zigbee) radio transceivers for the remaining 1s. During this period, the averaged noise data is transferred from all nodes to the gateway/server via a custom time division multiplexed area (TDMA) network protocol with self-configuring multi-hop routing. The data transfer to the gateway/server can be via internet local area network (LAN) or general packet radio service (GPRS) to the computer server, where the data is processed [22], see Figure 1.

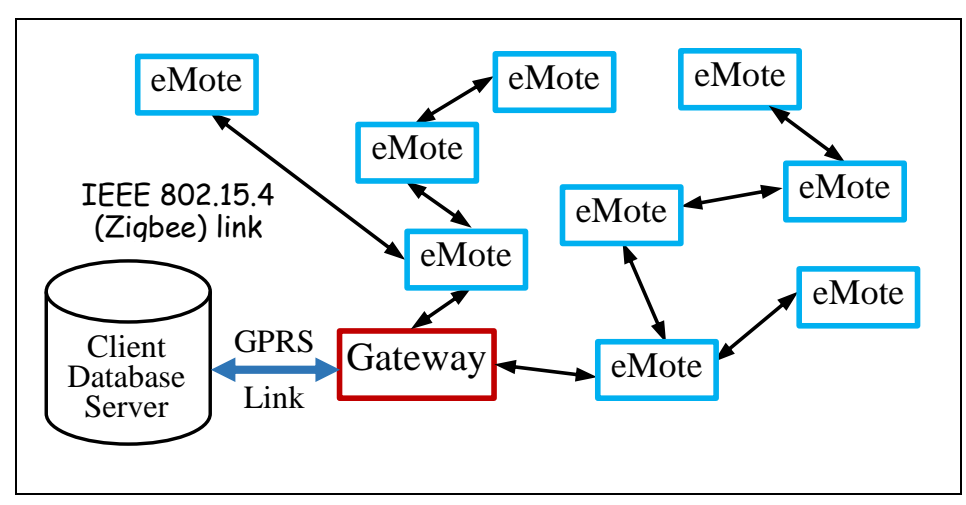

Figure 1 Data communication network

Up to 100 eMote units can be deployed in a single high-density mesh network, resulting in high spatial and temporal resolution noise data, in all weather conditions, with minimal maintenance requirements. The system is self-organized and robust for failing units and it can reconfigure easily. Furthermore, the system is flexible changing coverage on scale by adding or removing sensors within a network. A typical eMote deployment will site the units on lampposts at roadside locations, at approximately $2.5 \mathrm{~m}$ above ground level, to effectively monitor air quality and noise levels without the eMote system being vandalised. 
As this paper explores specifically the use of the eMote for noise measurement further relevant details of the components of the eMote system are now presented.

The eMote microphones, similar to those used in precision instruments, convert sound waves to electrical energy, generating a voltage in response to a certain acoustic pressure. This voltage is processed by applying appropriate A-weighting and integration schemes, to transform the reading into the desired $L_{\text {Aeq }}$ parameter. The eMote uses the omnidirectional microphone: AOM-6545P-R [28], which has an overall frequency response range of $20 \mathrm{~Hz}$ to $20 \mathrm{KHz}$ similar to that of human hearing. The microphone is located underneath the sensor system pointing downwards, see Figure 2.

The eMotes provide real-time, minute-by-minute noise data ( $L_{e q}$ in $\mathrm{dBA}$ ) over an approximate range of 50-94dBA. The system enables synchronized measurements at several monitoring points simultaneously. Their low cost, small size and ability to be flexibly deployed on existing urban street furniture (as shown in Figure 2) means that they potentially address the deficiencies in traditional monitoring approaches, as discussed in Section 1. The system monitoring continuously can operate for 1 year on an internal battery pack [22], or for up to at least 2 years when combined with a solar panel and rechargeable battery depending on availability of sunlight. The eMote units need to be placed facing direct sunlight in order to produce the maximum solar output. Once the battery pack is exhausted, the sensor operates intermittently on the rechargeable battery, depending on availability of solar radiation, for the lifetime of the solar panel. The energy consumption depends on the sampling rate, integration time and transmission schedule for the sensor data. The sensor system powers down when the processor unit and parts of the circuits are not in use to increase battery life. This increases the flexibility of deployment of such units, as power management and power consumption are one of the main issues with monitoring environmental noise over long periods. The gateway is the only unit that requires connection to a constant power supply, such as the UK mains system [22].
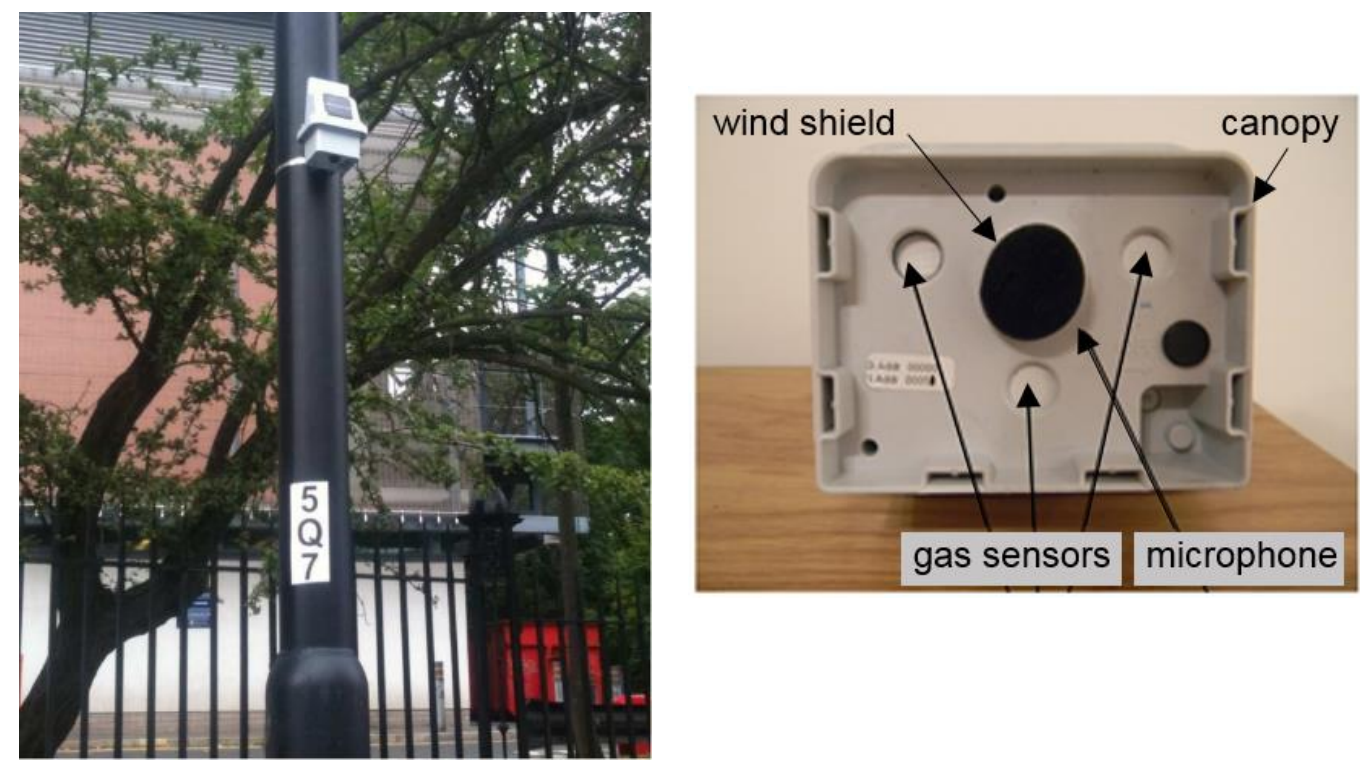

Figure 2 eMote mounted on a lamppost (left) and close up of sensor payload (right) [22]

\section{Performance of the eMote}

Three eMotes, with serial ID numbers 152, 162 and 164, were tested alongside two precision Brüel and Kjær, B\&K type 2260 sound level meters (SLMs). The eMotes were mounted in a quiet recording studio environment, at Newcastle University. Ambient noise in the laboratory was monitored as being less than 20dBA. In separate tests, the eMote units were subjected to white and pink noise sources. In white noise, different frequencies have the same power spectral density, a 3dBA increase in overall energy per octave band whilst in pink noise the power spectral density is inversely proportional to the frequency giving constant overall energy in each octave band [29]. Both types of noise are produced using a B\&K Type 1405 Noise Generator, with a broadband signal output in the range $0-20 \mathrm{kHz}$. The noise generator was connected to a B\&K Type 2706 Power Amplifier to amplify the output signals above $40 \mathrm{~dB}$. These two units, whilst not considered up-to-date technology, were tested and checked internally by Northumbria University, prior to being loaned for this study, and were considered adequate for the tasks required. 
The noise monitors were mounted at height $1.5 \mathrm{~m}$ above the floor level, capturing noise from a single Sony SS-H551 loudspeaker at a distance of $2 \mathrm{~m}$ away. Several tests were carried out with the source at different heights, in Figure 3 shown at $1.5 \mathrm{~m}$. However, the tests reported in this paper were at $0.5 \mathrm{~m}$ above the ground consistent with the reference height of noise modelled for traffic noise [30]. The microphones of the eMote units were faced downwards (consistent with their on-street deployment), whilst the precision sound level meter microphone was at grazing incidence (facing up), see Figure 3. The temperature in the recording studio averaged $21.8^{\circ} \mathrm{C} \pm 0.8^{\circ} \mathrm{C}$ throughout the monitoring period. This was demonstrated not to have a statistically significant effect on the response of the measurement system at $95 \%$ level of confidence.

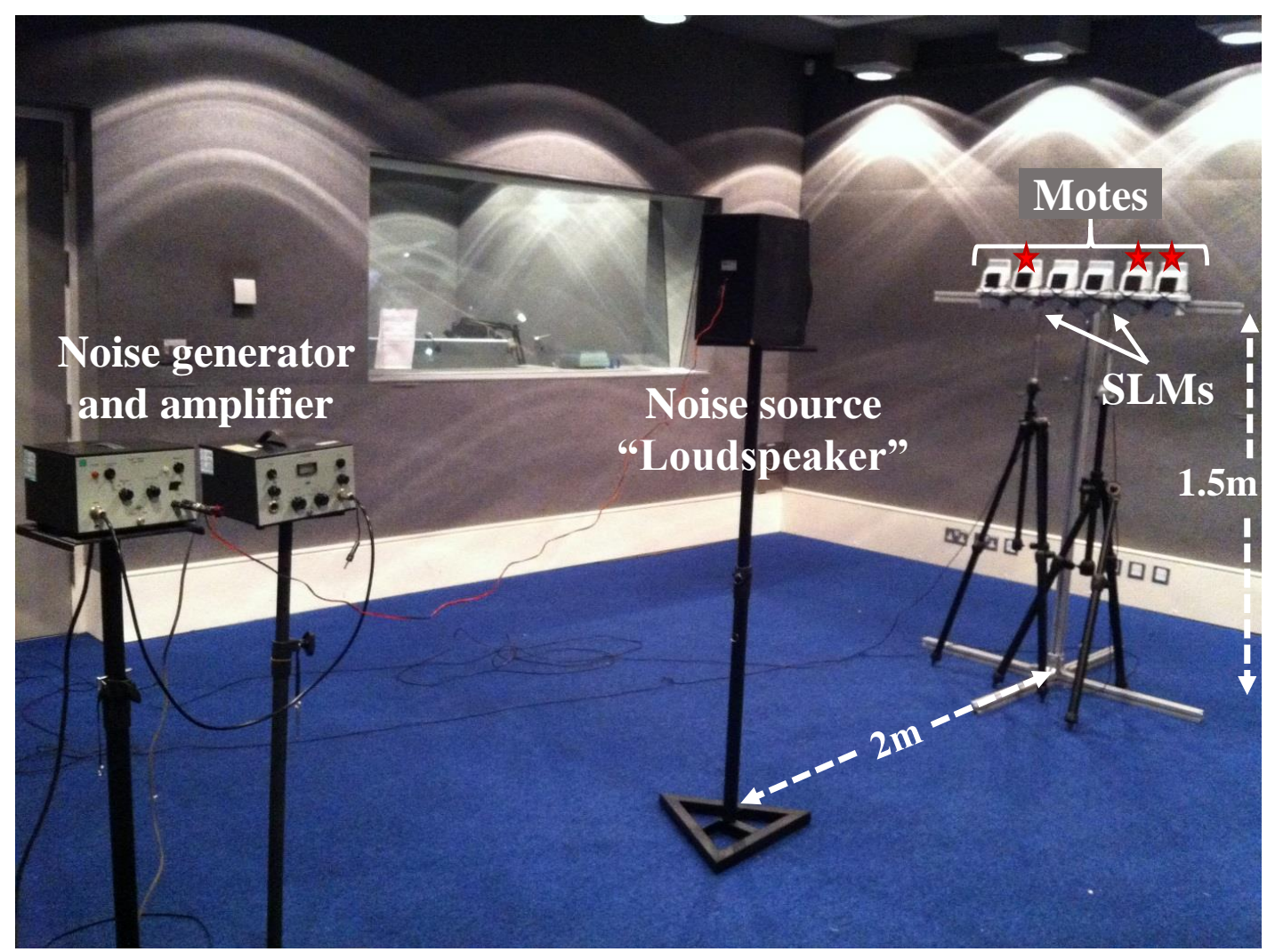

Figure 3 Experiment setup at indoor environment

Noise levels were varied over a range from 50dBA to 90dBA, in steps of $5 \mathrm{dBA}$ using white and repeated for pink generated noise source. The co-location was indoors in an acoustic environment known to be quieter by more than 10dBA so that the background did not contribute to the source noise level. Regression lines were plotted to compare the noise levels measured by the reference microphone on the SLM 2260 with the data from the three eMotes marked with red stars in Figure 3. The data set was fitted with a linear regression line separately for white and pink generated noise, and the combination of both. Three correction algorithms were developed, as shown in Figure 4.

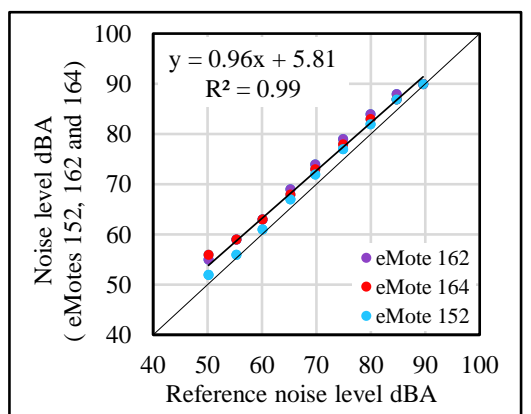

(a)

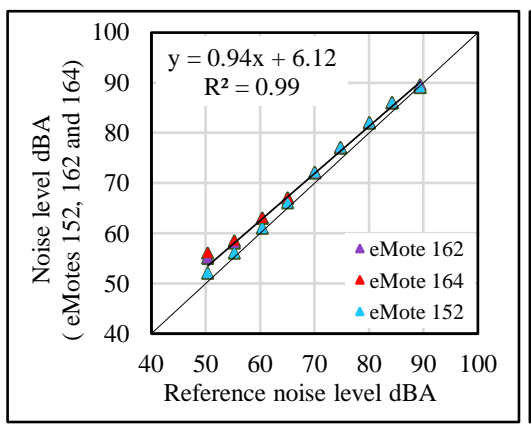

(b)

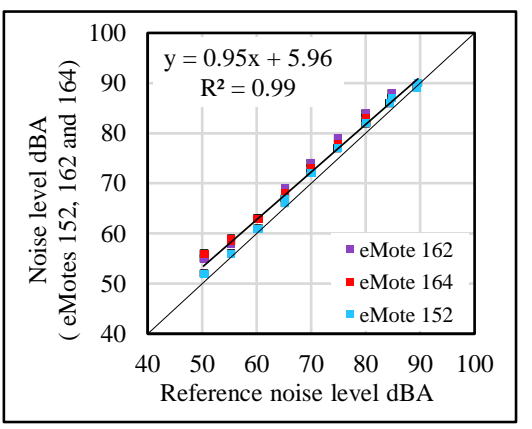

(c)

Figure 4 Correction models to adjust noise data from eMotes (a) white generated noise, (b) pink generated noise and (c) combine based on measurements made at $0.5 \mathrm{~m}$ 
Given the measurement limits of the eMotes, values below the lowest detection limit of each unit (measured as 53dBA, 55dBA and 51dBA respectively for units 162, 164 and 152) were excluded from the regression analyses. The results showed that in the range between (55-70)dBA the mean overestimation of $L_{\text {Aeq, } 1 \mathrm{~min}}$ measured by the eMote units was $3 \mathrm{dBA}$ for the white noise source, $2.3 \mathrm{dBA}$ for pink and 2.7dBA for combined, whilst, above 70dBA all three eMotes have almost identical response as the reference microphone. A student t-test was used to compare the slopes and intercepts of the regression lines for white, pink and combined noise at $95 \%$ confidence level, the calculated $p$ values were $>0.05$ indicating that there was no statistically significant differences between the regression coefficients at $95 \%$ confidence level. Given that the response of the microphone AOM6545P-R and associated circuitry is consistent for both white and pink generated noise it is concluded that the eMote units have similar response to the noise at low, medium and high frequencies. The regression equations for these eMotes, and their response to the coloured noise, form the basis of the correction algorithms. The next step was to demonstrate the transferability of the three algorithms derived in the laboratory to other eMotes when used out in the field.

\section{Outdoor Accuracy Test}

Outdoor monitoring was carried out in Newcastle, UK, at the roadside site adjacent to a major radial route (Jesmond Road), carrying high volumes of traffic, and may be considered one of the noisiest places in the city with respect to vehicular noise. Ten eMotes were collocated with the same SLM as used in section 3 , above a cabin which houses precision air pollution monitors, as part of the UK Automatic Urban and Rural Network (AURN) [31]. The eMotes deployed had been used in previous studies three years earlier and were refitted with new batteries but retained the same microphone. The installation at the Cradlewell AURN cabin on Jesmond Road was undertaken from 17th December 2015 to 9th February 2016. The cabin is situated on spare land, which runs parallel to the main road, terminating at a junction with another minor road, next to the cabin. The area to the north is lined with residential and commercial properties, whilst on the opposite side of the road there is green space, featuring a number of mature trees, in the grounds of a residential care home, see Figure 5.

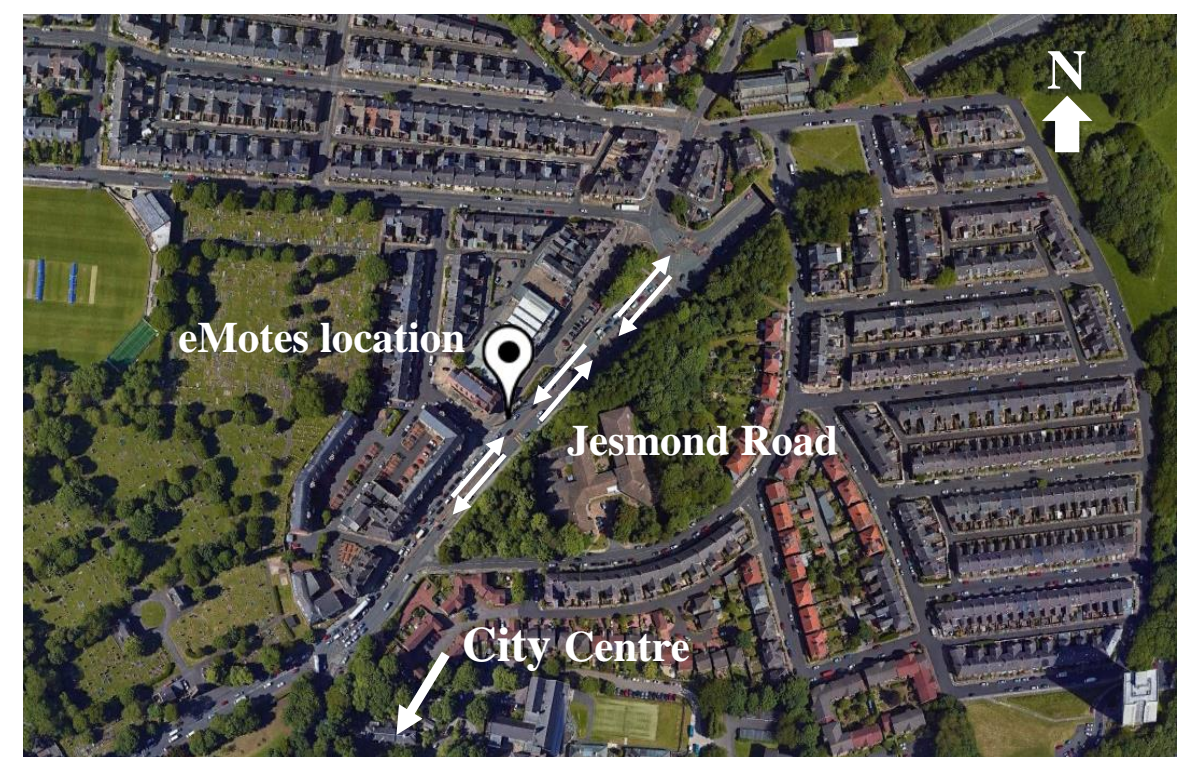

Figure 5 Location of the monitoring site at Cradlewell AURN cabin on Jesmond Road the A1058 North East Newcastle City Centre (Google maps)

\subsection{Network Setup}

Figure 6 shows the setup and position of nine eMotes, in a three-by-three matrix, on a steel mounting pole, alongside the microphone housing of the Type 2260 SLM. The microphone of the precision system was B\&K Type 4189 and installed facing upward at height $362 \mathrm{~cm}$ above ground level. The tenth eMote (unit ID 165) was installed above the microphone of the SLM. The differences in eMote positions are discussed further in the data comparison section of this paper.

The SLM was installed inside the AURN cabin, using its mains power supply and connected to the microphone by a $10 \mathrm{~m}$ long cable (B\&K AO 0442). The microphone of the SLM was protected with a windshield to minimise the effect of wind on the measurements. The instrument was setup to sample at $100 \mathrm{~Hz}$ and log broadband sound levels, $1 / 3^{\text {rd }}$ octave spectral levels, A-weighted LAeq, as well as 


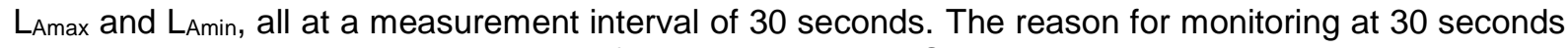
was to assist with the synchronisation of the eMote with the SLM data.

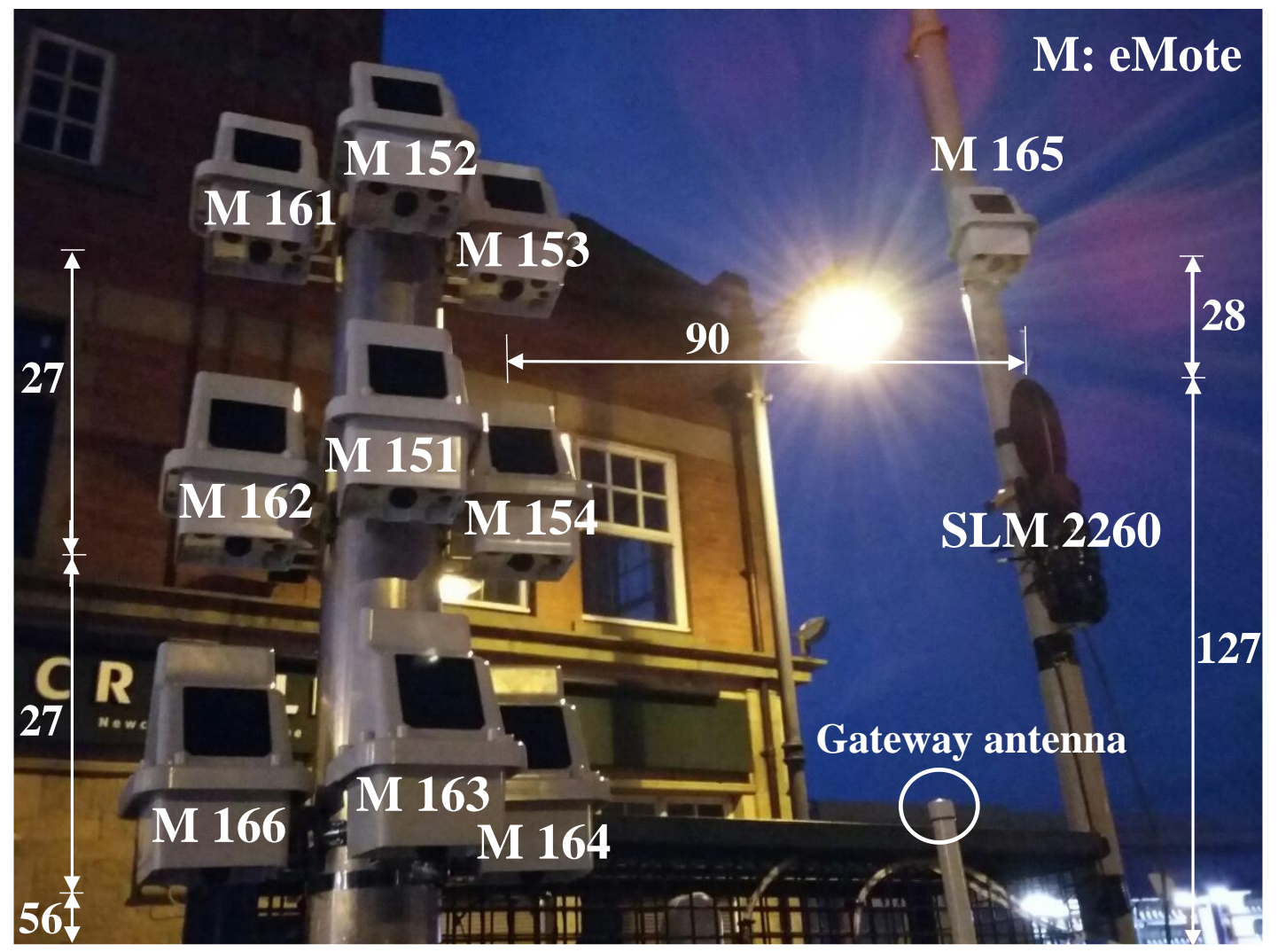

Figure 6 Experimental layout showing co-location of precision system and eMote units at the Cradlewell AURN cabin (distances shown in $\mathrm{cm}$ )

In order to overcome the limited capacity, noise data logged to the internal memory card in the SLM was transferred manually every two weeks to a laptop placed inside the cabin. Excessively loud, transient noise levels, exhibiting significant divergence from ambient background intermittently experienced at the site, were logged and recorded for manual classification and post-processing, to identify their source. These noise events were usually sirens. Previous research has used LAmax as the criteria for defining noise events [32], therefore the SLM also was setup to record on the PC, as audio files (.wav) any period with $L_{A m a x} \geq 85 \mathrm{dBA}$ with duration longer than 2 seconds. These events were time stamped and subsequently examined to identify the source, using a data processing module in the noise prediction model AVTUNE (Airviro Traffic and Urban Noise Evaluator) to display the audio files with their timestamp and equivalent, minimum and maximum noise levels [33]. A gateway was installed at the top of the cabin to receive and send the noise data from the ten eMotes directly to a server at Envirowatch Ltd., who then supplied the data to the University.

\subsection{Data Manipulation}

Data for the week $17 / 12 / 2015$ to $23 / 12 / 2015$ was used in this analysis. Before any analysis took place missing data records were removed from both the SLM and the eMotes. Time offsets between the eMotes and the SLM clock were determined manually. A correction of up to 1 to 3 minutes was applied to time-align paired noise levels between each eMote and the precision SLM. The data availability from eMote systems over the period was $88 \%$ on average, compared to $100 \%$ available from the precision system. Missing data was typically caused by a failure in data communication between the eMotes and the gateway in times of weak Wi-Fi signal. Before handling and analysing the collected data the noise levels from the sound level meter were time synchronised and averaged to one minute, to align with the noise levels provided by the eMote units. The open source statistical programming language ' $R$ ' [34] was used for manipulating and analysing the noise data.

\subsection{Environmental Noise $L_{\text {Aeq, } 1 \text { min: Precision System versus eMotes }}$}

The interquartile range of the LAeq, 1 min from the precision SLM noise level data at Jesmond Road was found to lie in a narrow band from 72.8 to $75.6 \mathrm{dBA}$. However, the data set had a high positive kurtosis, equal to 7.1 , reflecting the prevalence of outliers. The distribution of noise levels was non-normal, and 
right-skewed (skewness $=-0.26$ ). The minimum noise level recorded by the precision system was $51.4 \mathrm{dBA}$, which was below the lower limit of the measurement range of 6 out of 10 of the eMote units, whilst the maximum noise level was $92.2 \mathrm{dBA}$, close to the eMotes' upper measurement range. The noise levels at the site are consistently high, due to the traffic volumes, but peak noise events were found to be caused by the activities of specific vehicles, mainly sirens on emergency service vehicles that frequently use the road due to the close proximity of a hospital.

A box plot in Figure 7(a) was used to understand the variability in the noise levels, across all 10 eMotes, compared to the precision SLM and shows the median, first and third quartile, 95\% confidence limits and outliers. The three groups of red, green and blue boxes respectively, represent the eMotes at the lower, middle and upper rows on the post. The measurements were affected by the differences in location on the post of the microphones relative to the noise source from traffic, the flat roof of the cabin and the influence of the presence the other eMotes, causing both reflection and scattering of sound. In general, the median noise levels provided by the eMotes were higher than that from the precision system, particularly from those units which were installed at the upper row of the matrix. This may be expected given the better angle of-view of the direct source noise, and additional clearance from the diffracting edges of the AURN cabin. On the other hand, the other units in the lower and to a lesser extent the middle row in the matrix were placed closer to the top of the cabin surface, which presents a barrier between the direct noise path from the traffic stream and the receivers. The sky blue box in the plot represents the noise data recorded by the eMote with unit ID 165 , which was located above the precision system. Therefore, the measured differences are possibly due to the general over-estimation of levels by the eMotes, which was observed also in the indoor colocation exercise.

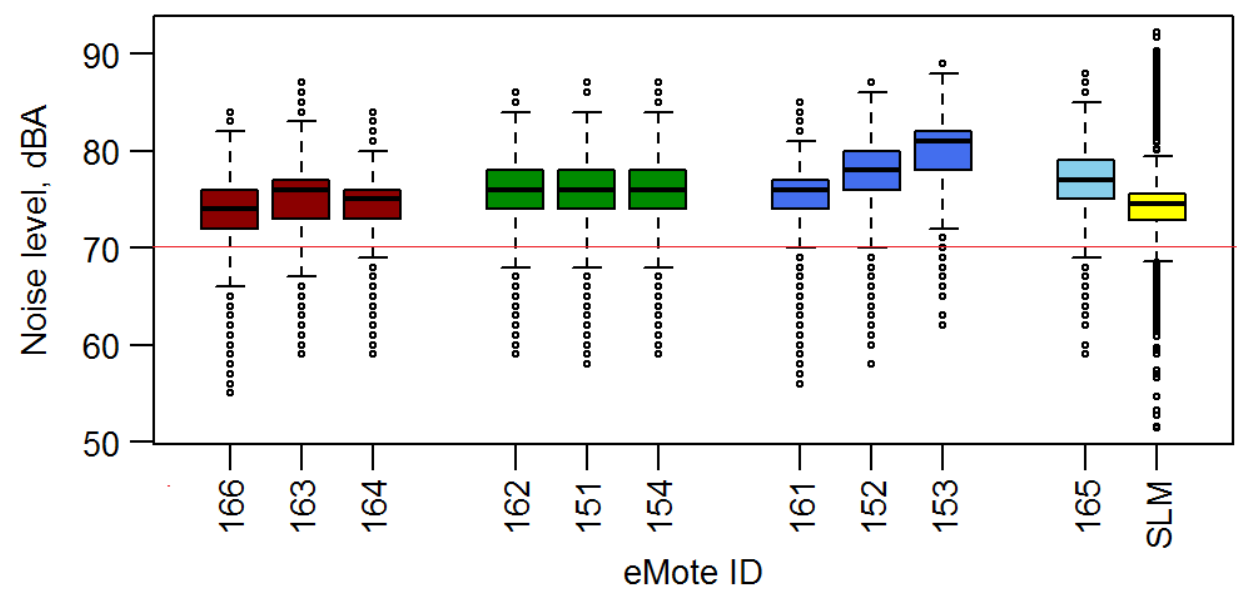

(a)

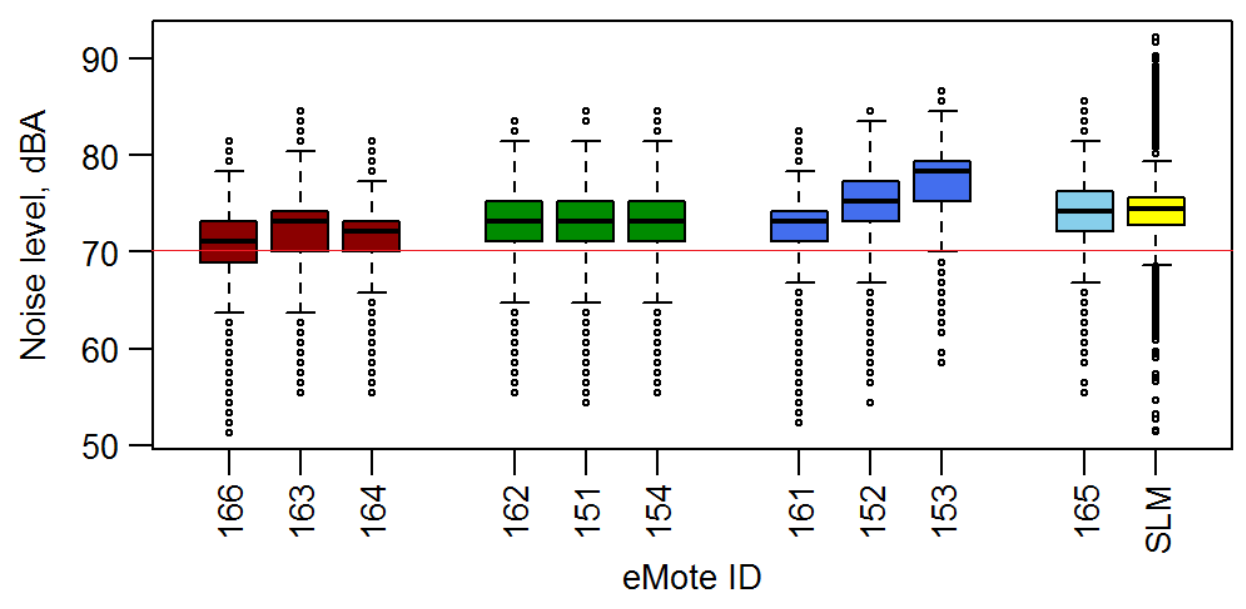

(b)

Figure 7 Box plots of $L_{A e q, 1 m i n}$ data at Jesmond Road, (a) without and (b) with corrections 
Therefore, the three correction algorithms (derived from white generated noise, pink generated noise and both combined) were applied separately to the recorded noise levels from the eMotes. The specific formula, which gave a better agreement between the eMotes and the precision system, was identified using the concordance correlation coefficient (ccc). According to Lin [35] the ccc is the best statistical parameter to investigate the degree of similarity between two instruments. It evaluates the degree to which pairs of data fall on the $Y=X$ line, it contains measurements of accuracy (how far the best-fit line deviates from the identical line) and precision (how far each observation deviated from the best-fit line). Usually the $c c c$ value ranges from 0 to 1 with $c c c=1$ representing the best possible agreement. It was found for example that the ccc value for the raw data from eMote 165 was 0.51 and it improved to $0.72,0.67$ and 0.71 when using the white, pink and combined equations respectively. Consistently the algorithm for white noise performed best and therefore was adopted as the generic correction algorithm for all eMotes.

The actual lower measurement threshold of eMotes was also found to be unit specific, falling in a range of around 50dBA to 55dBA. Therefore, all noise levels below 55dBA were ignored when applying the generic correction equation. Figure 7 (b) presents the corrected data for direct comparison with uncorrected in Figure 7(a). The median noise levels became more consistent with the sound level meter especially for eMote165 where the differences were just within 0.3dBA, due to their close proximity. On the other hand, the LAmax and levels close to the threshold as expected are less obviously improved because these lower levels are known to be less precisely measured as revealed in the indoor co-location exercises.

This underestimation at peak sound pressure levels partially is due to the manner in which the eMote processes the signal by sampling a smoothed voltage profile at $5 \mathrm{~Hz}$. This means the eMote does not respond to sharp increases in noise in the same way as does the SLM sampling at $100 \mathrm{~Hz}$.

\subsection{Agreement between eMotes and the Precision System at 1-Minute Resolution}

Figure 8 (a), (b) and (c) illustrates at one minute, the insensitivity of the eMote to measurement of peak transient events (i.e. sirens), and the lower threshold effect. The variation in noise is due to interrupted traffic flow because of cyclist and pedestrian movements crossing Jesmond Road, transients in vehicle flow; variation in fleet composition and bus stops at the Cradlewell AURN site. In Figure 8(a), the raw data from the eMote165 is presented, prior to the application of any correction factor. In Figure 8(b); correction with the white noise derived algorithm improves the ccc from 0.51 to 0.72 , showing better agreement between the precision measured and corrected noise levels. In Figure 8(c); peak events have been removed, and further improvements of the $\mathrm{ccc}$ and $\mathrm{R}^{2}$ values are noted. Similar results have been achieved for all of the remaining nine eMote units tested, see Table 1. Overall the $R^{2}$ and ccc values were increased after applying the correction equation and removing short loud events an exercise consistent with [36] given that these events were found not to be traffic related (sirens) and are outside the scope of any abatement measure. However, the ccc value for the eMotes 161, 163, 164 and 166 were decreased. This may be due to their position in the network with eMote 161 highest and furthest from the SLM and units 163, 164 and 166 installed at the lowest row of the matrix and in closest proximity to the top of the cabin and therefore, statistically this is a real effect and not due to the limitation of the correction factor. This is discussed further in section 4.6.

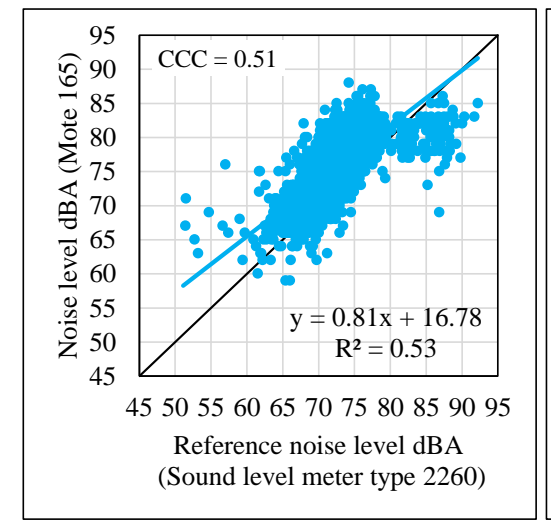

(a)

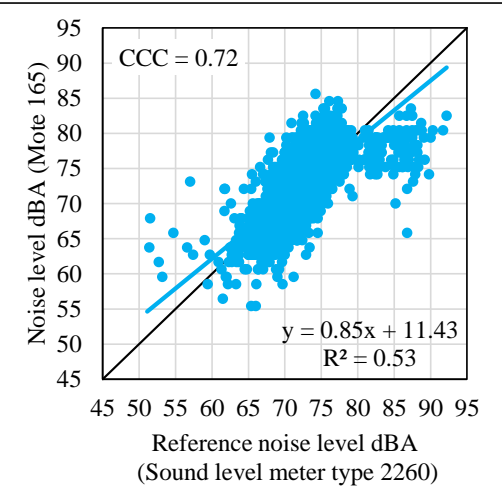

(b)

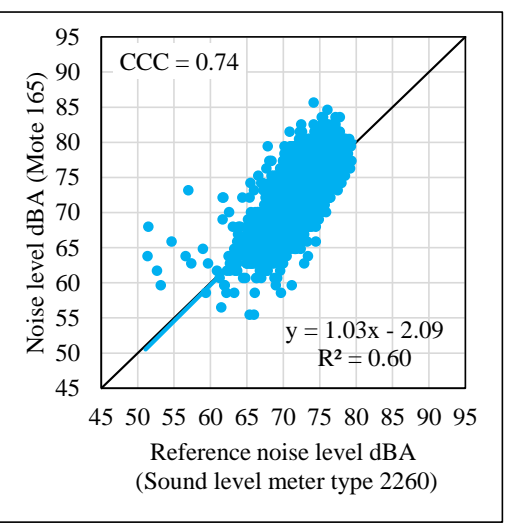

(c)

Figure 8 eMote165 vs. B\&K 2260, LAeq,1min data at Jesmond Road (a) before correction, (b) after correction and (c) with removed short duration event 


\begin{tabular}{l|ll|ll}
\hline \multirow{2}{*}{ eMote ID } & \multicolumn{2}{l|}{ R-square } & \multicolumn{2}{l}{ ccc } \\
\cline { 2 - 5 } & Before & After & Before & After \\
\hline 151 & 0.56 & 0.63 & 0.63 & 0.73 \\
152 & 0.57 & 0.65 & 0.46 & 0.74 \\
153 & 0.50 & 0.59 & 0.25 & 0.45 \\
154 & 0.51 & 0.57 & 0.60 & 0.70 \\
161 & 0.57 & 0.66 & 0.72 & 0.66 \\
162 & 0.57 & 0.65 & 0.65 & 0.73 \\
163 & 0.52 & 0.58 & 0.69 & 0.62 \\
164 & 0.59 & 0.67 & 0.77 & 0.54 \\
166 & 0.60 & 0.68 & 0.76 & 0.50 \\
\hline
\end{tabular}

Table 1 The R-square and ccc values for LAeq, 1 min from each eMote LAeq, 1 min in the network before and after correction and removal of short duration events

However, whilst $90 \%$ of the noise levels at Cradlewell AURN cabin site fell in the range 69.0 to 76.9dBA, levels which are generally within the eMotes' dynamic range, the sensors are overestimating noise towards the upper end of that range. Given that the eMote currently samples a smoothed curve at a frequency of $5 \mathrm{~Hz}$ rather than the "raw" signal at $100 \mathrm{~Hz}$ consistent with the sound level meter type 2260 , the units not only have a slower response to sudden and short changes in sound pressure level, the traffic noise fluctuations are not fully captured. Therefore, to improve the response to these shortterm variations in noise level, the way in which the voltage signal is processed needs to be changed and sampling rate increased.

Another issue to consider is the eMote sensors utilise an Analog to Digital Converter (ADC) with resolution of 10-bits, compared to the 24-bit ADC in the precision SLM. The eMote unit therefore provides a nominal dynamic range of $20 . \log _{10} 2^{10} \approx 60 \mathrm{~dB}$ [21], and divides the vertical range of a single sensing into $2^{10}$ discrete levels. However, practically, the least significant 1-2 bits for any ADC are noisy, which further reduces the effective dynamic range by approximately $10 \mathrm{~dB}$. In addition, the simple analog front-end circuitry used in the eMote noise sensor has a relatively high noise floor which further limits the effective dynamic range [37]. While increasing sampling rate provides better temporal resolution of the sensed data, resulting in a more precise measurement [38], this would require changes within the eMote electronic circuitry. Increasing the number of operations required for sensing (i.e. increasing sampling rate) would increase the power consumption of the unit, potentially to the detriment of long-term operation whilst on battery power.

\subsection{Agreement between eMotes and the Precision System at One-Hour Resolution}

Averaging data to hourly values reduces the influence of the short period events, which are more accurately measured by the precision system. The conversion of the one-minute data into hourly data smooths out much of the variation - quiet periods and loud periods within the hour tend to 'cancel each other out'. Consequently, as illustrated in Figure 9, the performance of eMote outputs compared to the precision system was improved by averaging the noise levels on an hourly basis. The ccc values were equal to 0.54 and 0.82 respectively before and after using generic correction equation. In the example, removal of the short duration loud events (b) versus (c) in the figure marginally improve the correlation between the eMote and the sound level meter (to $R^{2}=0.77$ ), but slightly decreases the degree of agreement $(\mathrm{ccc}=0.81)$. The performance of the remaining nine eMotes tested is illustrated in Table 2, the ccc values increased for all units except eMotes 152 and 153, this could again relate to their position in the matrix.

The z-test for the difference between two regression coefficients was used to test the null hypothesis that the coefficients (both intercept and slope) of the regression line between the sound level meter and each eMotes, using LAeq, 1 hour and LAeq, 1 min are statistically significantly similar, at the $95 \%$ level of confidence. The comparison was for three cases; before and after applying correction algorithm and for removing short events. The correct formula for this statistical test was explained in [39]. It was found at the $95 \%$ statistical confidence level, that the calculated $z$-values were not in the range of two standard deviations $( \pm 1.96)$ of the critical table values. Accordingly, there was evidence to reject the null hypothesis, and therefore, it was concluded that there was statistically significantly difference in the relationships between the precision system and eMotes, using noise data whether on a minute-byminute basis or hourly basis, see an example in Table 3 . 


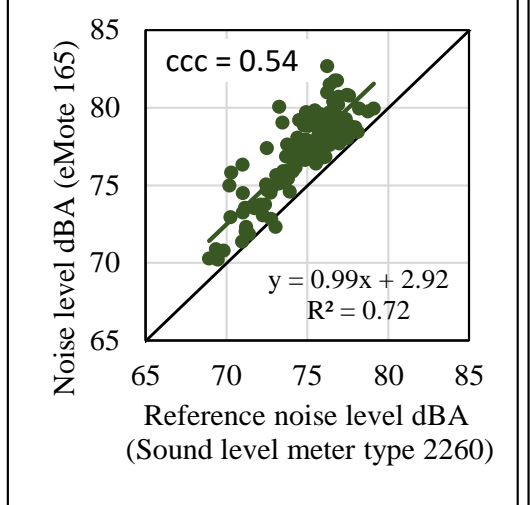

(a)

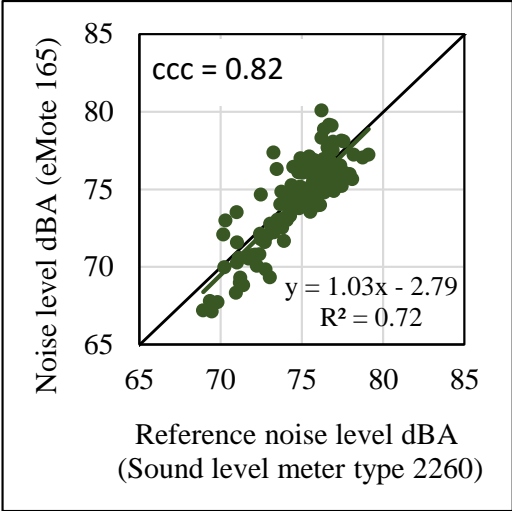

(b)

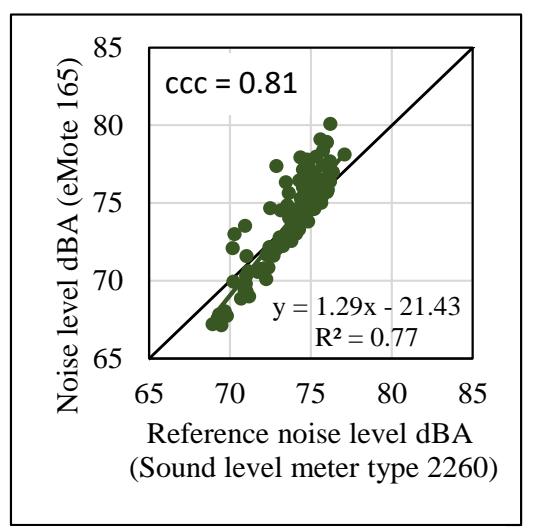

(c)

Figure 9 eMote165 vs. B\&K 2260_4 LAeq,1hour data at the Cradlewell AURN cabin (a) before correction, (b) after correction and (c) with removed short duration events

\begin{tabular}{l|ll|ll}
\hline \multirow{2}{*}{ eMote ID } & \multicolumn{3}{|l|}{ R-square } & \multicolumn{2}{l}{ ccc } \\
\cline { 2 - 5 } & Before & After & Before & After \\
\hline 151 & 0.76 & 0.81 & 0.74 & 0.80 \\
152 & 0.78 & 0.85 & 0.86 & 0.77 \\
153 & 0.68 & 0.76 & 0.48 & 0.36 \\
154 & 0.67 & 0.74 & 0.70 & 0.76 \\
161 & 0.78 & 0.85 & 0.60 & 0.67 \\
162 & 0.78 & 0.85 & 0.72 & 0.79 \\
163 & 0.71 & 0.76 & 0.57 & 0.63 \\
164 & 0.80 & 0.87 & 0.43 & 0.49 \\
166 & 0.81 & 0.89 & 0.41 & 0.45 \\
\hline
\end{tabular}

Table 2 The R-square and ccc values for $L_{\text {Aeq, } 1 \text { hour }}$ from each eMote $L_{\text {Aeq, } 1 \mathrm{~min}}$ in the network before and after correction and removal of short duration events

\begin{tabular}{|c|c|c|c|c|c|c|c|c|c|c|}
\hline \multirow[t]{3}{*}{ Noise data } & \multicolumn{4}{|c|}{1 Minute } & \multicolumn{4}{|c|}{1 Hour } & \multicolumn{2}{|l|}{$Z$ value } \\
\hline & \multicolumn{2}{|c|}{ Intercept } & \multicolumn{2}{|c|}{ Slope } & \multicolumn{2}{|c|}{ Intercept } & \multicolumn{2}{|l|}{ Slope } & \multirow[t]{2}{*}{ Intercept } & \multirow[t]{2}{*}{ Slope } \\
\hline & \multicolumn{2}{|c|}{ Coefficient SE } & \multicolumn{2}{|c|}{ Coefficient SE } & \multicolumn{2}{|c|}{ Coefficient SE } & \multicolumn{2}{|c|}{ Coefficient SE } & & \\
\hline Before correction & 16.8 & 0.68 & 0.81 & 0.01 & 2.92 & 4.02 & 0.99 & 0.05 & 3.4 & -3.53 \\
\hline After correction & 11.4 & 0.71 & 0.85 & 0.01 & -2.79 & 4.2 & 1.03 & 0.06 & 3.34 & -2.96 \\
\hline Removing loud events & -2.09 & 0.75 & 1.03 & 0.01 & -21.4 & 4.47 & 1.29 & 0.06 & 4.3 & -4.3 \\
\hline
\end{tabular}

Table 3 Comparing the coefficients of the regression lines between precision system and eMote165 for $L_{\text {Aeq, } 1 \text { min }}$ and $L_{\text {Aeq, } 1 \text { hour }}$

\subsection{Consistency of eMote Units}

Consistency in measurement performance across eMotes is important. However, the precise lower limit was found to vary with each unit: the lower limits for eMote 151, 152, 153, 154, 161, 162, 163, 164,165 and 166 respectively were found to be 52dBA, 50dBA, 53dBA, 53dBA, 51dBA, 53dBA, $51 \mathrm{dBA}, 55 \mathrm{dBA}, 52 \mathrm{dBA}$ and $50 \mathrm{dBA}$. Figure 10 presents a pairs plot showing the regression line between the paired measurements from the SLM and the ten individual eMotes in the network. The Rsquared values between each pair of eMote units was mostly higher than 0.95 , indicating a strong, positive linear relationship between unit results [40]. The variation of the regression model from the identical line was assessed using the ccc. The degree of the agreement between the eMotes measurements was wavering between substantial, moderate and poor, with ccc values varying mostly in the range between 0.5 and 0.99 according to the strength of agreement criteria presented in [41].

Interestingly the regression plots of eMote on eMote (see Figure 10) reveal three families of data, depending on the degree of scatter (low, medium and high) highlighted by yellow $\left(R^{2} \geq 0.98\right)$, green $\left(0.98>R^{2} \geq 0.95\right)$ and red $\left(R^{2}<0.95\right)$ colours. 


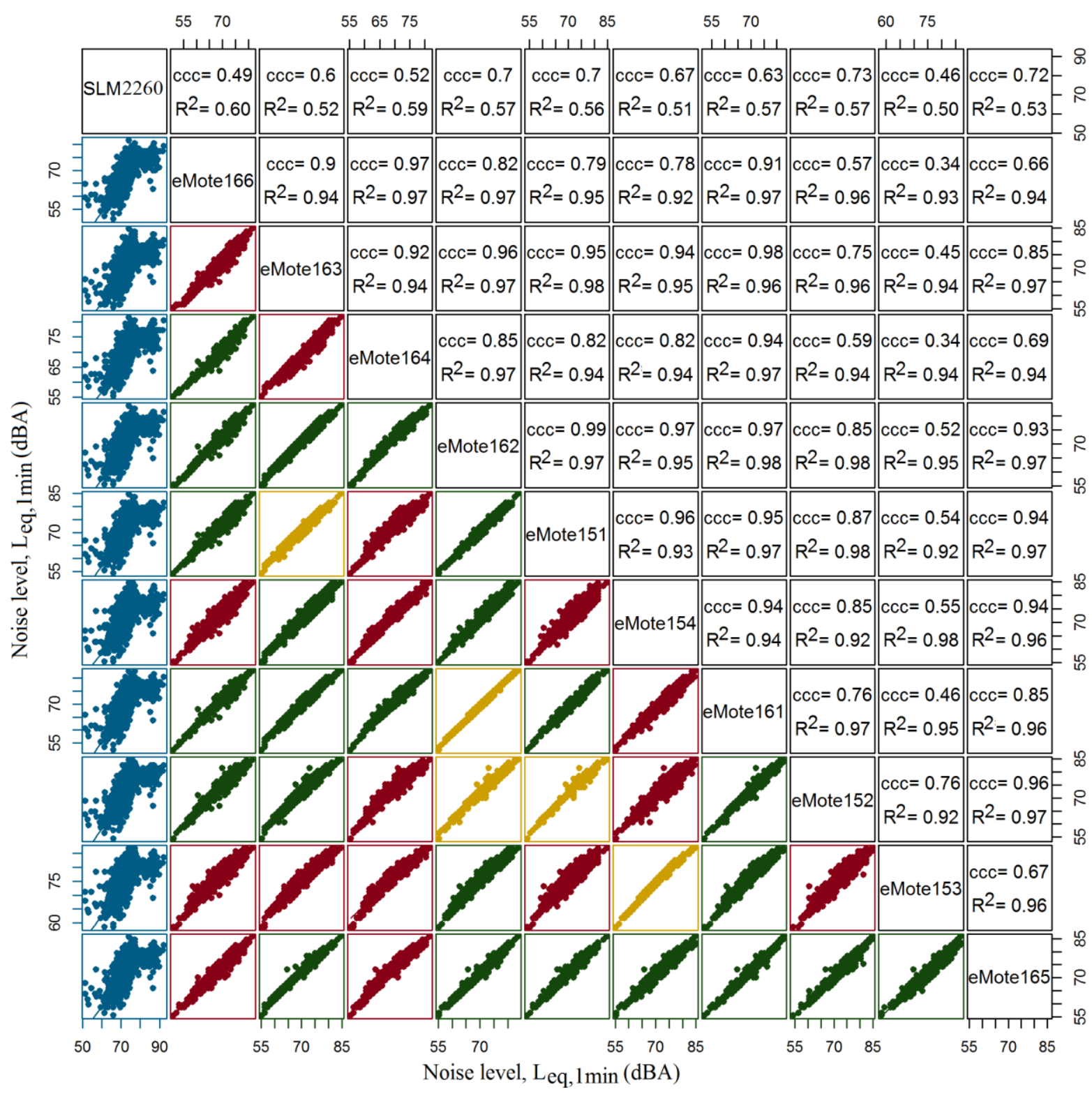

Figure $10 \mathrm{~A}$ matrix of plots represent the regression line between the SLM and the ten individual eMotes in the network, LAeq,1min raw data (corrected data and without removing short loud events)

The slight difference between the functionality of the individual units could be due to the difference in their positions during the measurement period, given each eMote in the network has different proximity to the top of the cabin and angle of view with the noise source from the road traffic. The less scattered points (yellow) appear between the units, which were installed above each other in the upper and medium row, whilst the plots (green) exhibit medium scatter which occurred between all eMote unit pairs installed within the matrix. The eMotes153, 154 and 164 positioned on the column of the matrix closest to the SLM showed high scatter (red colour) as with most other eMotes, particularly between those on the bottom row of the matrix where reflections and screening from the cabin were the highest. Also, eMote153 with lowest ccc values, revealed less agreement with other eMotes, consistent with the results of the co-location exercise of the wireless sensors indoors suggesting that this eMote consistently has a lower performance than other eMote sensors.

In all cases when compared with the precision SLM, eMotes exhibit lower $\mathrm{R}^{2}$ values typically in the range $0.50-0.60$ due to the detection range limitation, differences in sampling frequency, averaging period and location with respect to each other and the traffic source. It should be remembered that in outdoor co-location exercises there are actual variations in source noise levels as well as the "error of measurement" inherent in the eMote so differences in Figure 10 are a combination of both. 


\subsection{Influence of Meteorological Parameters}

One of the issues which arises with the microphones of noise monitors is their sensitivity to the changes in the weather conditions especially temperature, humidity, wind speed and wind direction, usually in outdoor measurements the microphones are provided with a windshield which is a special acoustic sponge to reduce the effects of wind-generated noise on the microphone diaphragm.

In this section the difference between the eMotes measurements relative to the precision system under several weather conditions was examined using the noise data from the first experimental setup. The meteorological conditions recorded by the UK Met Office were made available by the Newcastle City Council, for the monitoring periods (between the months of December 2015 and January 2016) from the closest meteorological recording station to the Cradlewell cabin on Jesmond Road. The station is deployed in Newcastle at the coordinate $\left(54^{\circ} 59^{\prime} 23.01^{\prime \prime} \mathrm{N}, 1^{\circ} 36^{\prime} 52.98^{\prime \prime} \mathrm{W}\right)$.

The meteorological dataset included many parameters at 10 minutes intervals. Therefore, the noise levels $L_{\text {Aeq, } 1 \mathrm{~min}}$ from each noise monitor were aggregated to 10 minutes and synchronised using the time stamps of the two datasets; noise levels and metrological parameters. Figure 11 presents the scatter plots between the difference of eMote data relative to the precision system against the measured on-site air temperature, relative humidity and wind speed for a period of one week (811 values), the noise data from the eMotes has been corrected with the algorithm based on white noise and removing loud non-traffic events. The difference between the eMote 165 and the reference sound level meter shows no statistically significant (at the $95 \%$ confidence level) dependence on temperature or relative humidity, see Figure $11(\mathrm{a})$ and (b). Across the similar measurement ranges the results here are not consistent with those of Van Renterghem et al. [15]. However, it must be recognised that the Van Renterghem et al. [15] deployment outdoors was for long term including winter and summer conditions, therefore the temperature was in the range $\left(-5^{\circ} \mathrm{C}\right.$ to $\left.30^{\circ} \mathrm{C}\right)$ and relative humidity $(30 \%$ to $100 \%)$.

Figure 11(c) shows dependency of the difference between eMote and precision measurements on wind speed, particularly starting from $1 \mathrm{~m} / \mathrm{s}$. The regression line with a slope and intercept equal to 0.94 and -1.63 respectively suggests a positive correlation between the difference obtained by the eMote relative to precision system and the wind speed.

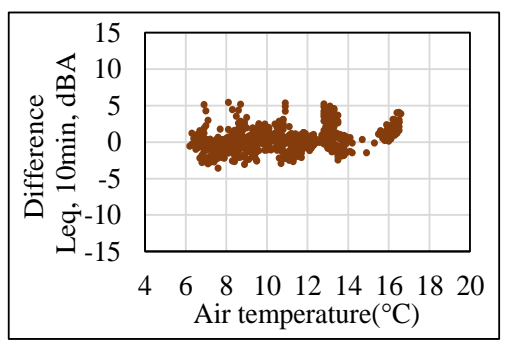

(a)

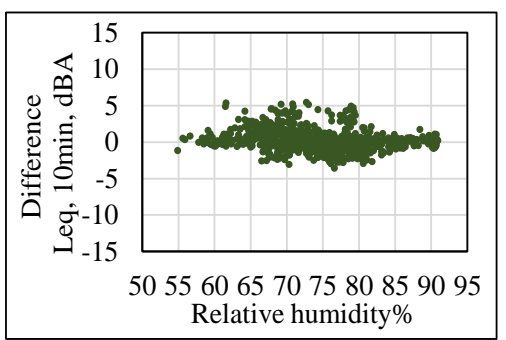

(b)

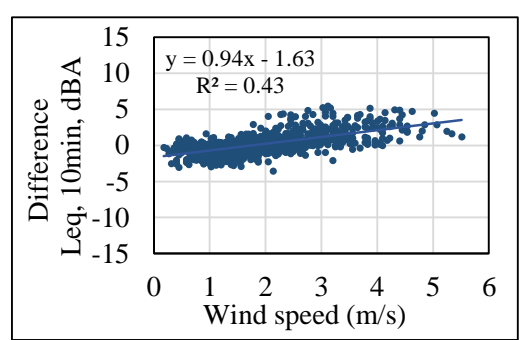

(c)

Figure 11 Scatter plots between ((a) air temperature, (b) relative humidity and (c) wind speed and the difference between eMote165 and B\&K sound level meter Type 2260

However, Figure 12 shows that the actual noise levels measured by the precision system 2260_4 are not showing any dependency on the wind speed, which was for $95 \%$ of the time, between 1 and $4 \mathrm{~m} / \mathrm{s}$.

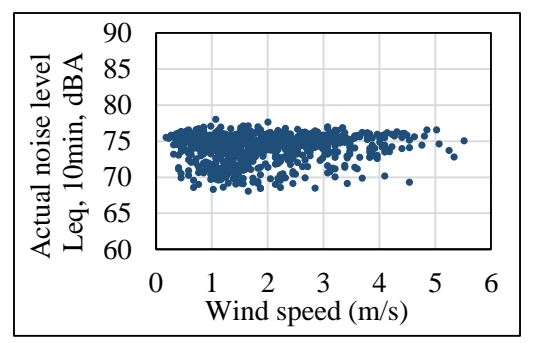

Figure 12 Scatter plot between the actual noise levels measured by precision B\&K 2260_4 and the magnitude of the on-site wind speed at Jesmond Road

Further investigation to understand precisely the reason for this observation in necessary; however, it is likely to be a combination of affects. Also, given the wind data was from a more remote location the 
interpretation of these results is limited. However, it is argued that wind refracts sound downwards producing reflections from the top of the cabin increasing the noise levels captured by the microphones facing downwards (eMote units) compared to the microphone facing up (SLM). In addition, the diffraction and refraction effects from the base of the eMote canopy (see Figure 2 right), due to its size is likely to only affect the highest of audible frequencies. Finally the more likely reason is the effectiveness of the acoustic wind shield for the microphone used in the eMote. The acoustic sponge covering the eMotes' microphone was limited in quality and may not have been sufficient to obviate the effects of wind upon microphone measurements. Besides according to the guidelines in the UK Calculation of Road Traffic Noise model (CRTN) [30] the noise measurements should only carried out with wind speeds of under $2 \mathrm{~m} / \mathrm{s}$ [42].

The behaviour of the remaining nine eMotes with different metrological parameters measured were consistent with that for eMote 165 above. Therefore, it is suggested that the eMote microphone itself is not unduly affected by wind in any different way to the SLM than reported here.

\section{Road Traffic Noise Evaluation}

In order to illustrate the potential value of the eMote as a tool for road traffic management, the eMotes were deployed to monitor road traffic noise in two further study areas namely Acorn Road and Gosforth High Street in Newcastle upon Tyne. These two sites, combined with Jesmond Road, represent a wide range of traffic conditions and road geometries providing an opportunity to demonstrate the value of the correction algorithms when applied to traffic noise data in different urban environments as well as giving a richer understanding of how the traffic flow patterns influence the associated noise level distributions across urban areas with different traffic control and/or management measures. Gosforth High Street is a major radial route, with a façade to façade distance of $(15-20) \mathrm{m}$, typically with a daily two-way traffic flow of $\approx 20,000 \mathrm{veh} /$ day, with numerous signalised intersections, to the north of the City Centre. The absence of bus lanes, the presence of car parking on both sides of the road, and loading and unloading for commercial premises, causes substantial congestion, and high, but also continually varying, levels of traffic noise. In contrast, Acorn Road is sited in a mixed residential-commercial area in Jesmond, Newcastle. It connects Osborne Road to St. George's Terrace. The road is $170 \mathrm{~m}$ long and about $11 \mathrm{~m}$ wide from façade to façade, and is lined on both sides with restaurants, banks and retail businesses. In 2014, at the time of this survey there was car-parking on both sides of the road, and the low levels of traffic (typically a daily flow of $3000 \mathrm{veh} /$ day) in the area are primarily due to the access requirements of local residential properties and to two small supermarket chain stores in the area.

Figure 13 shows histograms of the noise levels for all three study areas. The correction algorithm was tested not just across a range of traffic noise environments but also when adopting three different experimental approaches to the data collection in the on-street validation surveys. The first was based on simultaneous monitoring of precision with one co-located eMote over a period of 30 minutes conducted separately and twice at each of 24 sites at Gosforth High Street during the daytime from $7 \mathrm{am}$ to $7 \mathrm{pm}$; the second used simultaneous monitoring of precision monitor collocated with one eMote at one location at Acorn Road for 7hours during the day period from 7:00-19:00h and finally at one position continuously for one week at Jesmond Road including day, evening and night periods. The plots in the first column represent the precision noise data measured by the B\&K 2260 instrument, excluding the short, loud noise events (i.e. sirens). The histograms clearly demonstrate that the noise distributions are not normal. Anderson-Darling test was used to assess normality. At a statistical confidence level of $95 \%$ the $p$ values were less than 0.05 confirming a non-normal distribution [43]. In a normal distribution, the values for kurtosis and skewness are both equal to 0 [43], a kurtosis $>0$ indicates a "heavy-tailed" distribution, while kurtosis $<0$ indicates a "light tailed" distribution, skew to any side mean that the tail on that side is long relative to the tail on the other side. The variability in the environmental noise levels at the study areas was in a narrow range, where $50 \%$ of $L_{\text {Aeq, } 1 \text { min fluctuates }}$ within $\approx 3 \mathrm{dBA}$. The environment at $A$ corn $R$ Road with median $L_{\text {Aeq, } 1 \text { min }}=61.67 \mathrm{dBA}$ was found to be the quieter area with a few loud traffic events (not sirens) causing the tail at the higher end (skewness and kurtosis are +0.33 and +1.16 ) respectively. While, in Jesmond Road the noise levels are skewed toward the left of the graph, despite the much noisier environment with median LAeq, 1 min $=74.4 \mathrm{dBA}$, the traffic generates high levels of noise as a consequence of interrupted stop-start with noisy acceleration therefore, skewness and kurtosis was equal to -1.76 and +6.80 respectively. The distribution of noise data for Gosforth High Street exhibited a mix of quiet and noisy levels, median LAeq, $1 \mathrm{~min}=71.44 \mathrm{dBA}$ with small negative values for both kurtosis and skewness $(-0.24$ and -0.14$)$ respectively, again due to the continuously interrupted and unstable flow causing acceleration and deceleration throughout the day even when traffic flows fall between the peak hours. 
505560657075808590

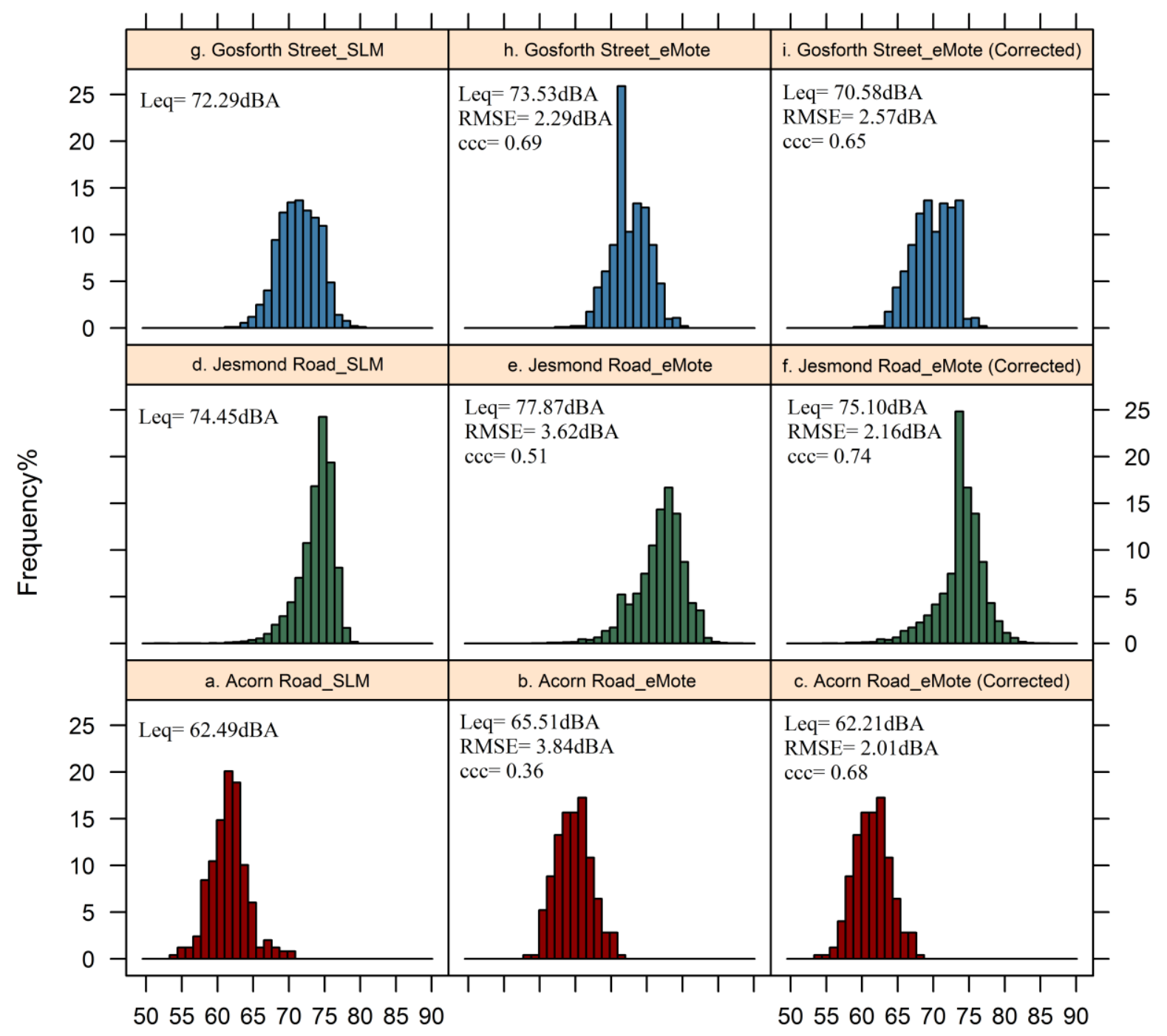

Noise level, $\mathrm{L}_{\text {eq, }}$ 1min $(\mathrm{dBA})$

Figure 13 Precision system (left) and eMote raw (middle), corrected (right) distributions of noise levels at the three urban study areas

The histograms in the second column in the figure represent the eMote noise levels (without correction), while the plots in the third column are for the corrected data. The LAeq,1 min, RMSE and ccc values were found for each case, it was obvious that using the three generic correction equation, especially the algorithm from white generated noise which is adopted in this study, improves the degree of agreement (ccc values) and decreases the error between eMote units and the precision system measurements. However, the case for Gosforth High Street was slightly different, where overestimated noise values were compensated by commensurate underestimated lower levels, resulting in less overall error in the raw data from the eMote units. Overall, the RMSE ranged between 2 and 2.6dBA, and always was less than 3dBA. Given the expected total allowable error for a sound level meter measuring steady broadband noise in a reverberant sound field is approximately $\pm 1.5 \mathrm{~dB}$ for a type 1 instrument and $\pm 2.3 \mathrm{~dB}$ for a type 2 instrument [44]. Furthermore, these error differences are barely detectable by the human ear, which is just sensitive to a change of noise level of $3 \mathrm{dBA}$, and it is generally accepted that changes of some $5 \mathrm{dBA}$ must be achieved to notice a significant change by the majority of the population [45], hence it can be concluded that at less than 3dBA the corrected eMote measured noise levels are of similar order of magnitude and not distinguishable by the human ear from the measurements made by the precision system. Thus demonstrating the value of the eMote as a tool for monitoring traffic noise related problems in urban areas.

This study presents sufficient evidence that the eMote units can be a promising tool for improving noise prediction and noise mapping for exposure giving noise level measurements of an acceptable 
level of accuracy in the range of (55 - 94)dBA. However, these units are not considered suitable to measure noise levels at night, when generally noise levels are low, due to their limitation to capture noise levels below 55dBA. Unfortunately this is often when noise pollution causes the most annoyance especially if levels cause sleep disturbance. However, eMotes would detect unacceptable sleep disturbance events exceeding 55dBA during quiet periods and therefore offer much potential as a screening measurement tool to be used prior to more detailed and expensive precision monitoring surveys in these quieter environments.

\section{Conclusion}

This paper demonstrated for the first time that due to consistent replicated systematic error observed in low cost noise sensors, measurements can be corrected using an empirically derived generic algorithm. Correction and validation exercises were carried out to verify and examine the relative performance of eMotes. The three algorithms resulting from an in-laboratory co-located exercise using white and pink noise sources, were found not to be statistically significantly different when used to correct the eMote data in the field. This suggests that the response of the AOM-6545P-R microphone is not dependent on frequency. However, when the eMote readings were systematically corrected the concordance correlation coefficient (ccc) for white noise source was consistently higher. Therefore, it is recommended that all eMote data were corrected using the white noise source correction algorithm. The wireless units showed a consistency of performance between themselves. The eMote is not sensitive to loud event noise and therefore automatically screens "non traffic" related noise, a requirement of the UK Defra guidelines for noise action planning, to address the management of noise issues and the effects from just road traffic noise [36]. However, in quiet noise environments the eMotes are only useful as a screening tool due to the lower noise floor $(\leq 55 \mathrm{dBA})$ limitation. This suggests that the eMote technology is useful for daytime measurements of highly trafficked roads and overnight to screen for sleep disturbance caused predominantly by heavy goods vehicles often using prohibited roads for deliveries.

The eMotes have a slow response to the sudden changes in the noise levels and all have a data capture range between 55dBA and 94dBA. The generated generic correction equation based on white noise source improved the agreement between the noise data from the units with the precision system, however the sensors long term deployments still were underestimating noise at the lower levels and therefore overestimating exposure if levels remain substantially below 55dBA. Although there were always missing data from the eMotes, the loss of data does not affect the precision of long-term deployments.

Overall, the results showed that low cost eMotes sensors provide data that is highly correlated to the reference microphone with an accuracy of better than 3dBA when compared to class 1 sound level meter. Also, eMotes can provide: (a) continuous monitoring of pollution hotspots; (b) a truer picture of the noise levels across an area during the periods when highly trafficked and levels across the eMote data capture range (55 - 94) dBA prevail; (c) a better understanding of the causes of noise when traffic, metrological conditions etc. are measured simultaneously and (d) opportunity to better measure the exposure of people spending substantial time in environments affected by traffic related noise given the one minute averaging continuously over long periods of time. Knowledge of the causes of excessive noise allows more effective action planning and improved policy formulation. eMotes also are a valuable tool to measure the impact of interventions introduced to reduce noise and a potential screening tool for sleep disturbance. The new technology offers high temporal and spatial coverage, which is recommended by the European regulation for the purpose of environmental noise mapping. This research has demonstrated the value of pervasive sensor technologies for monitoring and mapping noise levels $\geq 55 \mathrm{dBA}$ which are considered harmful when occurring consistently throughout the day as in major cities such as London, New Delhi, Beijing etc. with 24 hour economies. Finally, the generic equation consistently performed well for all 24 eMotes used in this study and the authors are of the opinion that the algorithm is appropriate to correct measurement using AOM-6545P-R microphone which showed no dependency with temperature over the range $\left(6^{\circ} \mathrm{C}-17^{\circ} \mathrm{C}\right)$ and humidity (55\%-91\%). Further validation of a random selection of the eMotes will be carried out in the future.

\section{Future Development}

The technology used in the eMotes is now ten years old and a higher spec microphone, which can easily be a hundred times more expensive is not necessarily hundred times more accurate as inferred by [15] could be considered. In addition, the associated circuitry employed the least expensive components that delivered useful data, and coupled with the limited energy budget, the design of the noise circuit was compromised to minimise power consumption. Given the advances in technology, a 
future designs of the electronics could deliver a much wider dynamic range and lower noise floor for a similar cost and power but the microphone is likely to be much the same. Also, possibilities of increasing sampling rate to $10 \mathrm{~Hz}$ and transfer of data averaged to shorter time periods (less than the current 60 seconds) are fairly straightforward with access to power. Depending on the stakeholder needs, to justify investment, new designs of more bespoke pervasive sensors for use in many other applications are being developed. For example in 2014, using the knowledge and experience gained from the eMote system, Envirowatch Ltd, UK, manufactured a new mote named the nMote which was designed specifically to monitor noise pollution within airports. The nMotes have a 50dBA range that can be adjusted to lie somewhere between the lower bound of $40 \mathrm{dBA}$ and the upper bound of $125 \mathrm{dBA}$. These new units are stand-alone with an extra-large battery which operates for six months, and use a low power built-in GPRS communications capability, where the noise data can be transferred directly to the central server without needing a gateway in between, this is further improving the ease of deployment.

\section{Acknowledgement}

The findings presented in this paper form part of a doctoral research scholarship sponsored by the Ministry of Higher Education and Scientific Research, Kurdistan Regional Government, Iraq, the first thanks and appreciation must go to them. Also, the authors wish to thank staff at Envirowatch Ltd. for their assistance during the validation procedure and installing the eMote units and Amey for providing the traffic and noise data at the evaluation study sites is recognised with much appreciation. Finally, we acknowledge the EPSRC for funding the eMote hardware and the data collection platform used in this research.

\section{References}

[1] Murphy E, King E. Environmental noise pollution: noise mapping, public health, and policy. 1st ed: Elsevier Inc.; 2014.

[2] WHO. Burden of disease from environmental noise. World Health Organisation: Copenhagen, Denmark; 2011.

[3] de Paiva Vianna KM, Cardoso MRA, Rodrigues RMC. Noise pollution and annoyance: An urban soundscapes study. Noise and Health. 2015;17:125.

[4] WHO. Prevention of noise-induced hearing loss. World Health Organisation: Geneva; 1997.

[5] Mitchell P. Speed and road traffic noise. UK Noise Association; 2009.

[6] Steinebach G, Guhathakurta S, Hagen H. Visualizing sustainable planning: Springer; 2009.

[7] Moela RD. The impact of traffic noise pollution on the population of strubensvalley in roodepoort. University of Johannesburg; 2011.

[8] Berglund B, Lindvall T, Schwela DH. Guidelines for community noise: World Health Organization; 1999.

[9] WHO. Night noise guidelines for Europe. World Health Organisation: Copenhagen, Denmark; 2009.

[10] Kivelä I, Hakala I. Area-based environmental noise measurements with a wireless sensor network. Euronoise 2015. Maastricht2015.

[11] Kurakula V. A GIS-based approach for 3D noise modelling using 3D city models. Enschede, Netherlands: MSc dissertation. International Institute for Geo-information Science and Earth Observation, Enschede, Netherlands; 2007.

[12] Maisonneuve N, Stevens M, Niessen ME, Steels L. NoiseTube: Measuring and mapping noise pollution with mobile phones. Information Technologies in Environmental Engineering: Springer; 2009. p. 215-28.

[13] Kivelä I, Gao C, Luomala J, Hakala I. Design of noise measurement sensor network: Networking and communication part. SENSORCOMM: 5th International Conference on Sensor Technologies and Applications France2011.

[14] Santini S, Vitaletti A. Wireless sensor networks for environmental noise monitoring. 2007.

[15] Van Renterghem T, Thomas P, Dominguez F, Dauwe S, Touhafi A, Dhoedt B, et al. On the ability of consumer electronics microphones for environmental noise monitoring. Journal of Environmental Monitoring. 2011;13:544-52.

[16] Basten T, Wessels P. An overview of sensor networks for environmental noise monitoring. The 21st International Congress on Sound and Vibration. Beijing, China2014. 
[17] Wang C, Chen G, Dong R, Wang H. Traffic noise monitoring and simulation research in Xiamen City based on the Environmental Internet of Things. International Journal of Sustainable Development \& World Ecology. 2013;20:248-53.

[18] Neasham J, Sharif B, Blythe P, Bell M. A pervasive sensor network for environmental and traffic monitoring 1st Smart Environment Interest Group Conference. Institute for Transport Studies: London, UK; 2007.

[19] Santini S, Ostermaier B, Vitaletti A. First experiences using wireless sensor networks for noise pollution monitoring. EuroSys 2008: Workshop on Real-World Wireless Sensor Glasgow, Scotland2008.

[20] Segura-Garcia J, Felici-Castell S, Perez-Solano JJ, Cobos M, Navarro JM. Low-cost alternatives for urban noise nuisance monitoring using wireless sensor networks. Sensors Journal, IEEE. 2015;15:836-44.

[21] Hakala I, Kivela I, Ihalainen J, Luomala J, Gao C. Design of low-cost noise measurement sensor network: Sensor function design. SENSORDEVICES: First International Conference on Sensor Device Technologies and Applications. Venice/Mestre, Italy2010.

[22] Galatioto F, Bell MC, Hill G. Understanding the characteristics of the microenvironments in urban street canyons through analysis of pollution measured using a novel pervasive sensor array. Environmental monitoring and assessment. 2014;186:7443-60.

[23] Hakala I, Tikkakoski M, Kivelä I. Wireless sensor network in environmental monitoring-case foxhouse. SENSORCOMM: 5th International Conference on Sensor Technologies and Applications. France2008.

[24] Gubbi J, Marusic S, Rao AS, Law YW, Palaniswami M. A pilot study of urban noise monitoring architecture using wireless sensor networks. 2nd International Conference on Advances in Computing, Communications and Informatics. Mysore, India2013.

[25] Bell MC, Galatioto F. Novel wireless pervasive sensor network to improve the understanding of noise in street canyons. Applied Acoustics. 2013;74:169-80.

[26] Goodman PS. Internal report ITS. 2008.

[27] Hodges N, Bell MC, Galatioto F, Hill G, Neasham J, Suresh V. Sustainable network managementthe integration of intelligent transport systems and grid enabled pervasive sensors. Proceeding of 16th ITS World Congress. Stockholm2009.

[28] Mouser Electronics. 1964.

[29] Carter B. Op amp noise theory and applications. Op amps for everyone. Texas Instruments, Dallas2001.

[30] DoT. Calculation of road traffic noise. Department of Transport-Welsh Office, HMSO, London; 1988.

[31] DEFRA. Newcastle cradlewell roadside information. Department for Environment Food \& Rural Affairs ,; 2017.

[32] Yip CW. A study of the road traffic noise problem in Tsuen Wan, Hong Kong: MSc dissertation. Chinese University, Hong Kong; 1997.

[33] Goodman P, Bell M, Hodges N. The AVTUNE noise model. 11th International Symposium on Transport and Air Pollution2002.

[34] Crawley MJ. The R Book: John Wiley \& Sons; 2013.

[35] Lin LK. A concordance correlation coefficient to evaluate reproducibility. Biometrics. 1989;45:255-68.

[36] DEFRA. Draft noise action plan: Roads (including major roads) environmental noise (England) regulations 2006, as amended. London, UK: Department for Environment Food and Rural Affairs; 2013.

[37] Neasham J. Personal Communication. 2016.

[38] Akyildiz IF, Vuran MC. Wireless sensor networks: John Wiley \& Sons; 2010.

[39] Paternoster R, Brame R, Mazerolle P, Piquero A. Using the correct statistical test for the equality of regression coefficients. Criminology. 1998;36:859-66. 
[40] Ratner B. The correlation coefficient: Its values range between \&plus; $1 /-1$, or do they\&quest. Journal of targeting, measurement and analysis for marketing. 2009;17:139-42.

[41] McBride GB. A proposal for strength-of-agreement criteria for Lin's concordance correlation coefficient. Ministry of Health, National Istitute of Water \& Atmospheric Research Ltd, New Zealand; 2005.

[42] Goodman PS. The prediction of road traffic noise in urban areas: PhD thesis. Institute for Transport Studies, University of Leeds, UK; 2001.

[43] Minitab Ltd. The Anderson-Darling statistic. 2016.

[44] Kardous CA, Shaw PB. Evaluation of smartphone sound measurement applications (apps) using external microphones-A follow-up study. Journal of the Acoustical Society of America. 2016;140:327-33.

[45] Department of Transport and Main Roads Queensland Government. Volume 1: Road traffic noise 2016. 